\section{Military Technical College Kobry El-Kobbah, Cairo, Egypt.}

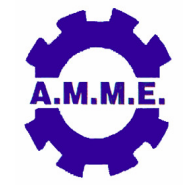

\title{
FLY AROUND MISSION IN NON- KEPLERIAN ORBIT
}

$15^{\text {th }}$ International Conference
on Applied Mechanics and
Mechanical Engineering.
A. Badawy*

\begin{abstract}
On-orbit manoeuvring in Low earth non-Keplerian orbit is discussed. High performance control strategies are required for these types of formation flying missions. The simple solution for Keplerian orbit is somewhat far from accuracy, while accurate solution for non-Keplerian orbit is not simple. Motion planning algorithms are used herein to accomplish successful fly around of orbiting objects. Close manoeuvres are performed as these manoeuvring objects approach the central body around which they will fly.
\end{abstract}

\section{KEY WORDS}

Space robotics, ISS, superquadric, potential field method, quaternions, motion planning.

\footnotetext{
* Egyptian Armed Forces.
} 


\section{INTRODUCTION}

The equations of motion of manoeuvring objects moving under a sole action of attraction force of a point mass planet is known as two-body point mass model, Keplerian orbit. Solution for this problem is well studied through Newton's law of gravitation and Kepler's law of orbits. Two-body point mass model gives fare approximation for actual orbit mechanics, whereas in some orbital mission this approximation should be enhanced considering perturbations [1].

For Low earth orbits (LEO) in which the orbit perigee height is below $1000 \mathrm{~km}$, the atmospheric drag effect becomes increasingly important. Drag is of non-conservative nature, and then continuous energy loss is produced. But because of Kepler's law the orbit becomes more circular in each revolution. A spiral inward orbit is finally produced with increasing orbit velocity until decay.

The rate of orbital decay depends on many parameters: orbital parameters such as height, and on ballistic coefficient which takes the shape and weight into account and on atmospheric density; which varies with time and geographic position. The difficult part of drag prediction is modelling of the atmosphere density.

The atmosphere density is related to solar flux, day-to-night. The solar flux has an 11-year cycle and the 27-day cycle caused by the extreme ultraviolet radiation $[2,3,4]$.

On-orbit manoeuvre control strategy based on potential field theory is discussed. The idea of potential field is first adopted for robot motion planning by Khatib [5]. The control strategy is based on establishing two distinct types of potential fields: attractive and repulsive. The first potential guides the manoeuvring agent toward its destination through providing a global minimum or attraction well. Whereas, the second potential counts for providing a protection system against collision between manoeuvring agent and all objects exist in space.

Satellite constellations reconfiguration problems for point mass model have been considered using potential functions [6, 7, 8, 9]. Autonomous on-orbit assembly for three dimensional model have been discussed using superquadric artificial potential fields $[10,11]$.

\section{KEPELRIAN ORBITS}

Keplerian orbits are governed by Newton's law of gravitation with only two particles of masses $m_{1}$ and $m_{2}$, distance $r$ apart. In restricted two-body problem, the mass $m_{1}$ is the principal mass where $m_{1} \gg m_{2}$. Principal mass experiences minute acceleration due to the existence of secondary mass, $m_{2}$. Consequently principal mass centre is considered as the origin of the inertial frame of reference. The two masses are attracted toward each other by gravitational force $F$ described as:

$$
F=\frac{G m_{1} m_{2}}{r^{2}}
$$


where $G$ is the universal gravitational const, assuming $m_{1}$ as Earth mass and $m_{2}$ as the manoeuvring object mass, $m$, so the gravitational force is then expressed as:

$$
F=\frac{\mu m}{r^{2}}
$$

where $\mu=3.986 \times 10^{14} \mathrm{~m}^{3} / \mathrm{s}^{2}$ is the Earth gravity constant, the maneuvering object acceleration is:

$$
\ddot{r}=\frac{\mu}{r^{2}}
$$

And is directed toward the Earth and is expressed in vector form as:

$$
\ddot{\mathbf{r}}=-\frac{\mu}{r^{3}} \mathbf{r}
$$

where $\mathbf{r}$ is the position vector directed outward from the primary body. Equation (4) expresses the linearized (Keplerian) orbital motion without perturbation.

\section{NON-KEPELRIAN ORBITS}

Part or all of Keplerian orbit assumptions are negated; therefore the simple straight forward model is not adequate. Forces other than gravity are in place due to various causes. This category of forces has a general description as perturbations. The main perturbations are due to Earth's oblateness, third body effect, solar radiation pressure, electromagnetic drag, and atmospheric drag. The decision about including one or more of these perturbations is mainly a function of maneuvering object and its orbit. Considering LEO, atmospheric drag is substantial.

A crucial part in drag effect prediction and control maneuvering object when considering drag, is the drag prediction and modeling. The upper atmosphere model is not accurate as many factors are in place such as the earth's day-night cycle, seasonal tilt, and variable solar distance, fluctuation in the earth's magnetic field, the suns 27 -day rotation and the 11-year sun spot cycle. Considering the perturbation effect as [12]:

$$
\ddot{\mathbf{r}}=-\frac{\mu}{r^{3}} \mathbf{r}+\mathbf{F}\left(\mathbf{r}_{o}, t\right)
$$

\section{Frames of References}

A straight beam of length $I$, and cross sectional area $A$, is places in LEO of altitude about $300 \mathrm{~km}$. Since the maneuvering object is moving in air, it is common to define two frames of references: body frame and wind frame. Both are centered at the mass center, $c$, of the maneuvering beam. The body frame of reference with its $x$-axis coincides with the beam longitudinal axis, $z$-axis directed downward, and $y$-axis completes the orthogonal set, obeying the right hand rule as in Fig. 1. 


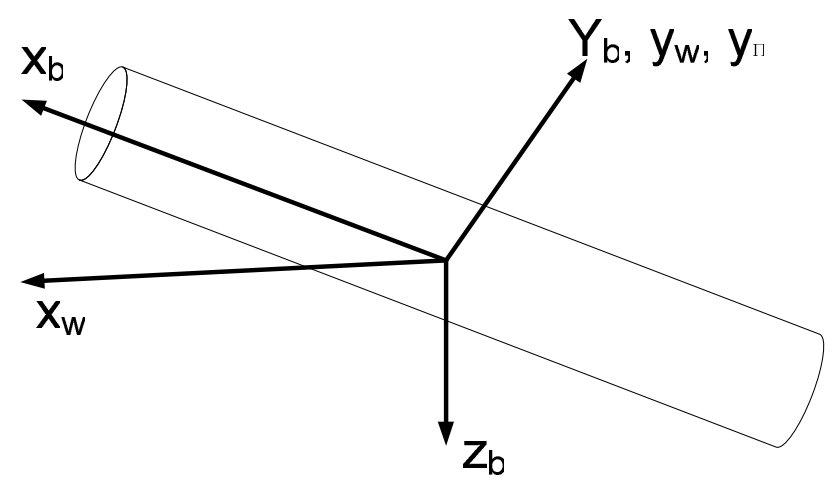

Fig. 1 Body frame of reference.

Wind frame of reference has its $x$-axis in the direction of velocity of the mass centre. Since the beam is symmetric about $x_{b}-y_{b}$ and $x_{b}-z_{b}$ planes, it is possible to define $a$ new body reference frame, $\Pi$, centered at the mass centre of the manoeuvring beam, such that its wind frame is obtained by rotating $\Pi$ about $y_{b}$-axis with a positive angle of attack $\alpha$. The positive direction of the angle of attack according to the defined orthogonal frames is in clockwise direction. The $x_{\Pi}-$ axis is chosen to be coincident with the $x_{b}$-axis, whereas the $z_{\Pi}$ - axis is chosen such that its positive part contains the component of velocity that is not on the $x_{\Pi}$ - axis. This implies that the unit vector in $z_{\Pi}$ - axis is expressed as:

$$
k_{\Pi}=\left(v_{y}^{2}+v_{z}^{2}\right)^{-0.5}\left\lceil\begin{array}{lll}
0 & v_{y} & v_{z}
\end{array}\right\rceil^{T}
$$

where

$$
{ }^{b} v_{c}=\left[\begin{array}{lll}
v_{x} & v_{y} & v_{z}
\end{array}\right]^{T}
$$

The rotation matrix of frame 'b' with respect to frame $\Pi$ is expressed as:

$$
{ }_{b}^{\Pi} \mathbf{R}=\left[\begin{array}{lll}
{ }^{b} i_{\Pi} & { }^{b} j_{\Pi} & { }^{b} k_{\Pi}
\end{array}\right]^{-1}
$$

The maneuvering beam velocity with respect to frame $\Pi$ is then expressed by:

$$
{ }^{\Pi} \mathbf{v}_{c}={ }_{b}^{\Pi} \mathbf{R}^{b} \mathbf{v}_{c}
$$

According to the previous definition of frame $\Pi$, the velocity of the center point has no component in $\mathrm{y}_{\Pi}$ direction, and then the angle of attack between the wind frame $\mathrm{w}$ and $\Pi$ frame is expressed as:

$$
\alpha=\tan ^{-1}\left(\frac{{ }^{\Pi} \mathbf{v}_{c}(3)}{{ }^{\Pi} \mathbf{v}_{c}(1)}\right) \in[0, \pi]
$$

And, finally the rotation matrix is expressed as: 


$$
{ }_{w}^{\Pi} \mathbf{R}=\left[\begin{array}{ccc}
\cos (\alpha) & 0 & -\sin (\alpha) \\
0 & 1 & 0 \\
\sin (\alpha) & 0 & \cos (\alpha)
\end{array}\right]
$$

\section{Lift and Drag Forces}

Forces acting on flying object are expressed by means of wind frame as:

$$
{ }^{w} \mathbf{F}=-\frac{1}{2} \rho_{a i r} A^{b} \mathbf{v}_{c}^{T b} \mathbf{v}_{c}^{T}\left\lceil C_{D} \quad 0 \quad C_{L}\right\rceil^{T}
$$

Where
$\rho_{\text {air }} \ldots$ Air density at orbit altitude.
A ... reference surface area.
$\mathrm{C}_{\mathrm{D}} \ldots$... Drag coefficient.
$\mathrm{C}_{\mathrm{L}} \ldots$... Lift Coefficient.

Drag and lift coefficients depend mainly on shape, speed, wind frame direction, and Reynolds number. These coefficients are usually determined experimentally. The point of application of drag force is expressed as:

$$
x_{c p}(\alpha)=0.124+0.243 \alpha-0.5 l
$$

Finally, aerodynamic force in body frame of reference is defined as:

$$
{ }^{b} \mathbf{F}={ }_{\Pi}^{b} \mathbf{R}_{w}^{\Pi} \mathbf{R}^{w} \mathbf{F}
$$

\section{Potential Field Definition}

This idea has been adopted and used in the motion planning algorithms for manipulators and robots as the artificial potential field method [5]. The potential filed is constructed through combining attractive and repulsive potential. Attractive potential functions to drive the manoeuvring object toward some destination configuration. While, repulsive potential serves to protect object from collision. The potential filed apply on object $i$ is a modified version from that defined as [10]:

$$
\mathrm{V}_{i}=\frac{C_{1}}{2}\left|\mathbf{P}_{i}-\mathbf{P}_{G, i}\right|^{2}+\frac{C_{2}}{2} \dot{\mathbf{P}} . \dot{\mathbf{P}}+\frac{C_{3}}{2} \overline{\mathbf{q}}_{i}^{T} \overline{\mathbf{q}}_{i}+\frac{C_{4}}{2} \boldsymbol{\omega}_{i}{ }^{T} \mathbf{I}_{i} \boldsymbol{\omega}_{i}+\sum_{j=1, j \neq i}^{m} A_{i} \frac{e^{-\alpha d_{i, j}\left(a, \mathbf{P}_{i}, \mathbf{P}_{j}\right)}}{d_{i, j}\left(\mathbf{a}, \mathbf{P}_{i}, \mathbf{P}_{j}\right)}
$$

where

$\begin{array}{ccll}\mathbf{P} & \ldots & \text { Object position vector. } \\ \mathbf{P}_{\mathrm{G}} & \ldots & \text { Goal point position vector. } \\ \dot{\mathbf{P}} & & \ldots & \text { Object velocity vector. } \\ C_{i} & \ldots & \text { Control gains. }\end{array}$




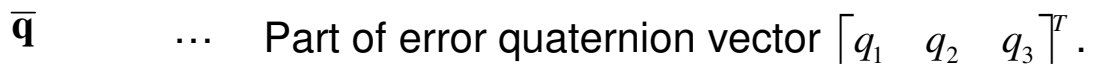

( $\quad \cdots \quad$ Object angular velocity vector.

I $\quad \cdots \quad$ Object inertia tensor.

A $\quad \cdots \quad$ Repulsive potential amplitude.

$\alpha \quad \ldots \quad$ Obstacle potential shape parameter.

$d \quad \cdots \quad$ Object separation distance.

a $\quad \cdots \quad$ Superquadric parameter vector $\left[\begin{array}{lllll}a & b & c & \varepsilon_{1} & \mathcal{E}_{2}\end{array}\right\rceil^{T}$.

$m \quad$... Total number of manoeuvring objects.

In order to guarantee global stability according to Lyapunov's instability theorem in the presence of drag force, the time derivative of the proposed potential field function should be negative definite since the proposed function itself is positive definite $[13,14]$. Object acceleration definition satisfies this condition is defined as:

$$
\ddot{\mathbf{P}}_{i}=-\frac{C_{1}}{C_{2}}\left(\mathbf{P}_{i}-\mathbf{P}_{i, G}\right)-C_{5} \dot{\mathbf{P}}_{i}-C_{6} \mathbf{F}_{i}{ }^{1}+\sum_{j=1, j \neq i}^{m} \nabla\left(A_{i} \frac{e^{-\alpha d_{i, j}\left(\mathbf{a}, \mathbf{P}_{i}, \mathbf{P}_{j}\right)}}{d_{i, j}\left(\mathbf{a}, \mathbf{P}_{i}, \mathbf{P}_{j}\right)}\right)
$$

where

$\mathbf{F}_{i}{ }^{1} \quad \ldots \quad$ Object drag force vector per unit mass.

$$
\nabla=\lceil\partial / \partial x \quad \partial / \partial y \quad \partial / \partial z\rceil^{T}
$$

Since the point of application of the resultant lift and drag forces does not coincide with the mass centre, these forces produce moment about the mass centre. The object angular acceleration is then defined as:

$$
\begin{gathered}
\dot{\boldsymbol{\omega}}_{i}=-\mathbf{I}^{-1}\left(C_{3} q_{i, 4} \overline{\mathbf{q}}_{i}+C_{4} \boldsymbol{\omega}_{i}+\boldsymbol{\omega}_{i} \times \mathbf{I} \boldsymbol{\omega}_{i}+\mathbf{T}^{1}+\sum_{j=1, j \neq i}^{m} \frac{\partial \mathrm{V}_{o b s_{i, j}}}{\partial d_{i, j}} \nabla^{q} d_{i, j}\right) \\
\nabla^{q}=\left\lceil\partial / \partial q_{1} \quad \partial / \partial q_{2} \quad \partial / \partial q_{3}\right\rceil^{T}
\end{gathered}
$$

where

$\mathbf{T}_{i}^{1} \quad \ldots \quad$ Object drag moment vector per unit mass. 


\section{RESULTS AND ANALYSIS}

Manoeuvre results for one and two orbiting satellites are shown in Fig. 2. Both objects start with the same orientation as goal configuration. The chosen orbit has an altitude of around $300 \mathrm{~km}$, with atmospheric density slightly higher than the average density and equals $5 \times 10^{-11} \mathrm{~kg} / \mathrm{m}^{3}$.

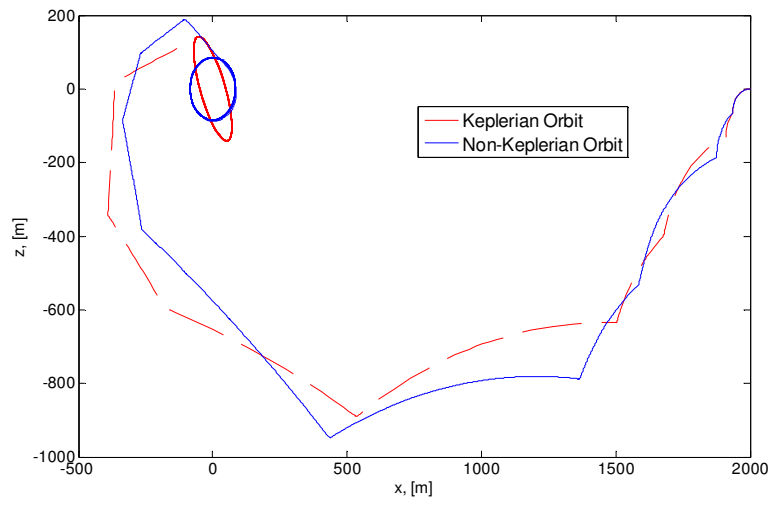

(a)

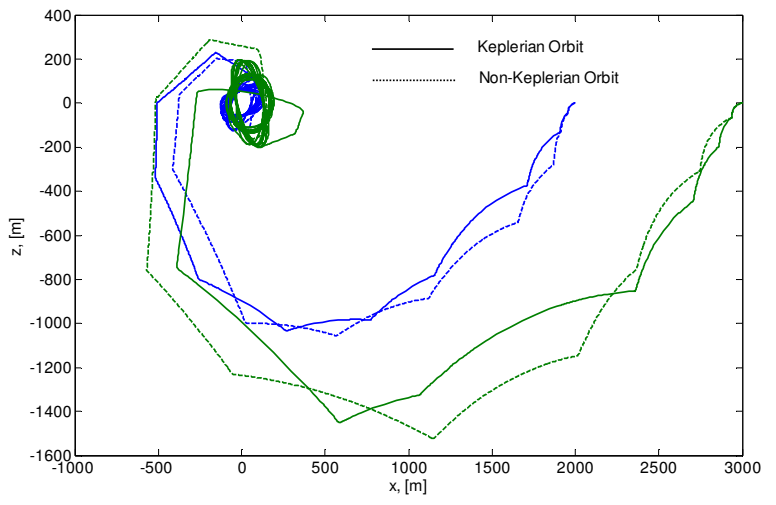

(b)

Fig. 2. Satellites in fly around manoeuvre.

In both figures, successful manoeuvres are performed. Satellite starts from an initial configuration at a distance of $2 \mathrm{~km}$ in $x$-direction from the goal position which is chosen, with loss of generality, at the origin of the local orbiting coordinate system. Non-Keplerian orbit motion planning shows better fly around goal points. By virtue of limiting controller interventions whenever loosing a suitable value of rate of approaching goal configuration, it is possible to utilize the orbital natural motion to reduce fuel consumption.

In Fig. 2-b, two satellites were launched to orbit the same body starting for different positions, but with the same orientations as those of goal configurations. Still and all both reach the desired goal configuration at which mutual repulsion occurs resulting in some level of distortion as they become close to each other, at goal position.

In Fig. 3-a rotational manoeuvre challenge is added. The initial configuration of the satellite is oriented $90^{\circ}$ about the z-axis, as shown in Fig. 3-b. In Fig. 3-c, angular velocities are shown in the first 100 seconds during which the first three terms in the error quaternions reach zero, so a match between satellite orientation and goal one. Velocity in the main direction of motion is illustrated in Fig. 3-d. The sudden change in satellite velocity is due to controller intervention.

A three dimensional manoeuvre is shown in Fig. 4. The initial orientation of the satellite is directed $90^{\circ}$ about the $x$-axis relative to the goal one. It takes just 80 second to reach goal orientation as the inertia about the axis is not as much as that for $y$ and $z$ axes. 


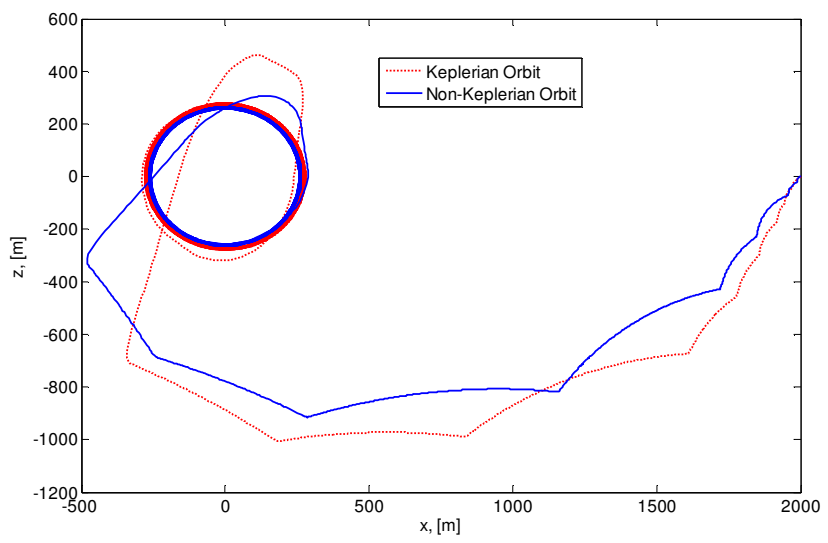

(a)

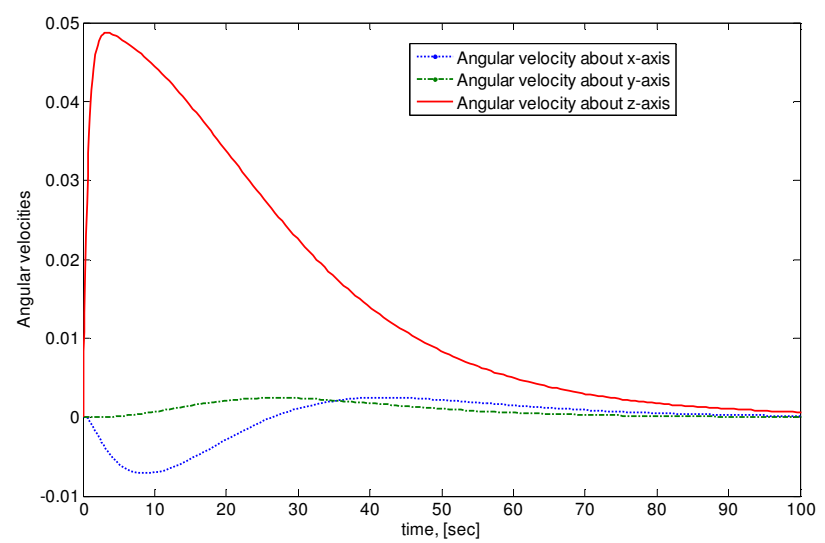

(c)

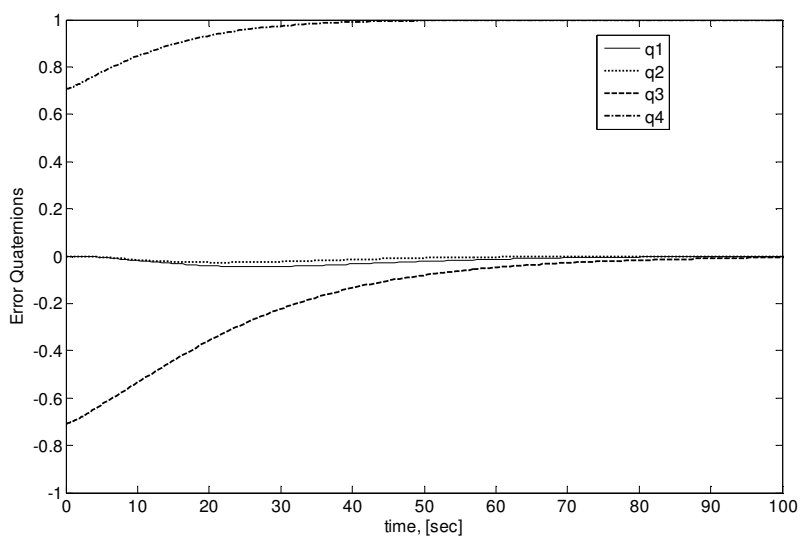

(b)

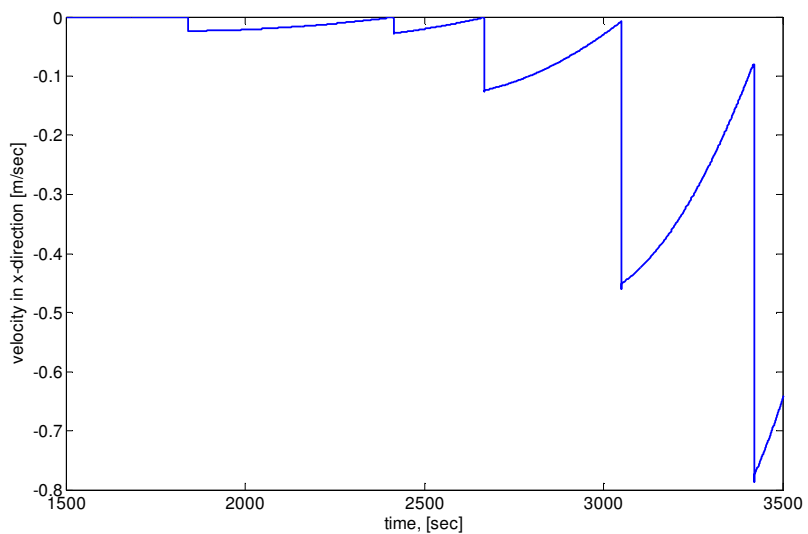

(d)

Fig. 3. Satellites in fly around manoeuvre (translation and rotation).
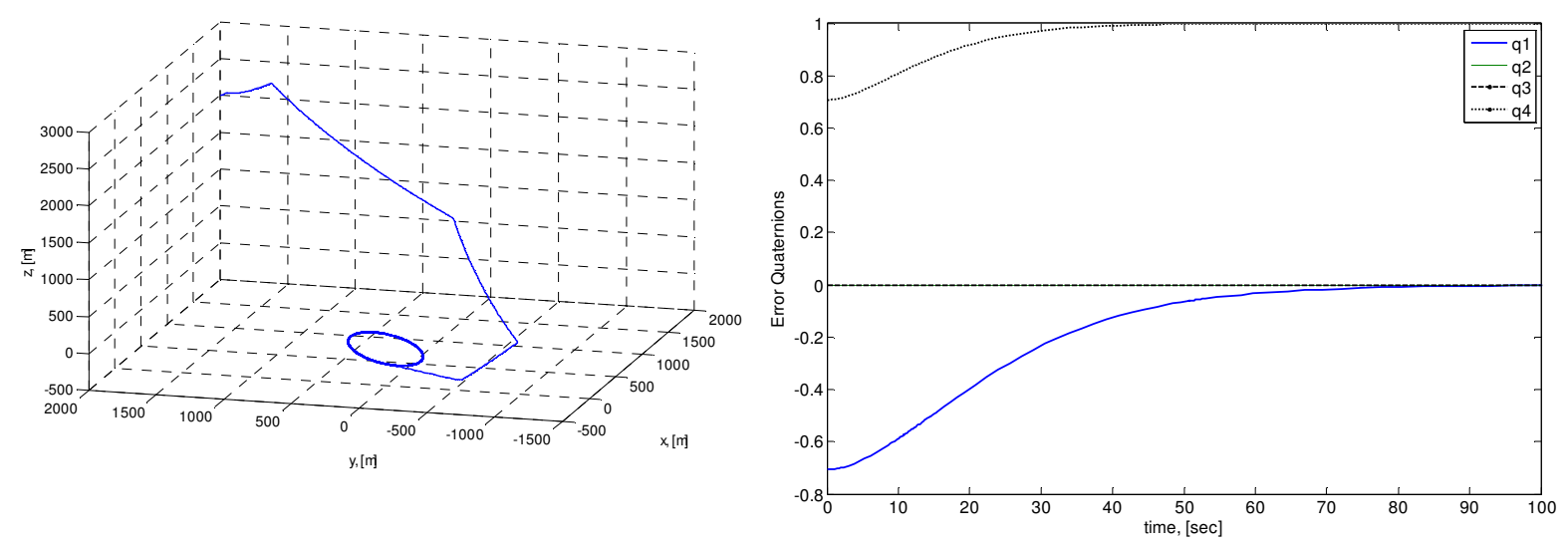

Fig. 4. Satellites in fly around manoeuvre (three dimensions).

\section{CONCLUSIONS}

The proposed method proves its ability to perform successful fly around mission in low earth orbit. Both two and three dimensional manoeuvres are successful despite initial or final configurations. The effect of drag force has a potential effect on the possible motion of the satellite. Performing this mission with two satellites is also 
successful utilizing the mutual repulsion between manoeuvring satellites. NonKeplerian orbit motion planning shows better fly around goal points than that of two point mass model since the existence of the continuous drag effect leads to more controller interventions, more energy consumed therefore.

\section{REFERENCES}

[1] Kane T.R., Levinson D.A., "Dynamics Theory and Applications", McGraw-Hill Book Company, 1985, ch. 6.

[2] AIAA Eduuction Series, "Orbital Mechanics", 1991, ch 9.

[3] Midshipman John L, "Determination of Atmospheric Density in Low-Earth Orbit Using GPS Data", Trident Scholar project report; no. 287, 2001.

[4] James G. Miller, "Atmospheric Density Model Errors and Variations in the Ballistic Coefficients", AAS/ AIAA, Quebic, Canada, 2001.

[5] Khatib O., "Real-Time Obstacle Avoidance for Manipulators and Mobile Robots", the International Journal of Robotics Research, 1986.

[6] Mclnnes, C.R. "Autonomous proximity maneuvering using artificial potential functions". ESA Journal, Vol. 17, 1993, 159-169.

[7] McQuade, F., Ward, R., Ortix, F., and Mclnnes, C.R., "The Autonomous Configuration of Satellite Formations using Generic Potential Functions", 3rd International Workshop on Satellite Constellations and Formation-Flying, Pisa, Italy, February, 2003.

[8] Izzo D., Pettazzi L., and Ayre M., "Mission Concept for Autonomous on Orbit Assembly of a Large Reflector in Space", IAC-05-D1.4.03, 56 ${ }^{\text {th }}$ International Astronautical Congress, Fukoka, Japan, September, 2005.

[9] Izzo, D., \& Pettazi, L. "Autonomous and distributed motion planning for satellite swarm". Journal of Guidance, Control, and Dynamics, 30 (2), 2007, 449-459.

[10] Badawy A. \& McInnes C.R. "On-Orbit Assembly Using Potential Fields", Journal of Guidance, Control, and Dynamics, AIAA, Vol 31, no 1, 2008, pp. 30-43.

[11] Badawy A. \& Mclnnes C.R. "Spacecraft Formation-Flying Using Potential Functions", Acta Astronautica, vol. 65, 2009, pp. 1783-1788.

[12] Tsuda, Y. and Scheeres, D., "State transition matrix approximation using a generalized averaging method", Journal of guidance, control, and dynamics, vol 32, no 6, 2009, pp 1781-1794.

[13] Csáki, F., "Modern control theories: nonlinear optimal and adaptive system". Akadémiai Kiadó Budapest, 1972, Chapter 5.

[14] Sastry, S. "Nonlinear systems: analysis, stability, and control". Springer-Verlag, New York, 1999. 